\title{
INEQUALITY IN ACCESS TO HEARING CARE FOR OLDER ADULTS IN RESIDENTIAL HOMES
}

Dr WHITE, JD.* 1, Speech and Hearing Sciences, Queen Margaret University, Edinburgh, EH21 6UU, United Kingdom.

Dr JOHNSON, C. 2, Speech and Hearing Sciences, Queen Margaret University, Edinburgh, EH21 6UU, United Kingdom.

Dr DEPLACIDO, CG. 3, Speech and Hearing Sciences, Queen Margaret University, Edinburgh, EH21 6UU, United Kingdom.

Dr MATTHEWS, B. 4, Speech and Hearing Sciences, Queen Margaret University, Edinburgh, EH21 6UU, United Kingdom.

STEENKAMP, EH. 5, Speech and Hearing Sciences, Queen Margaret University, Edinburgh, EH21 6UU, United Kingdom.

*Corresponding author:

Dr Joanna White, Email: jwhite@qmu.ac.uk

Tel: +44 (0131) 474 0000; Fax: +44 (0131) 4740001

Keywords: Older Adults, hearing loss, residential home, access inequality, aural rehabilitation, hearing assessment, staff training

For submission: Journal of Public Health 
Abstract

Background. The population of older people in residential homes is projected to rise. There are unrecognised hearing difficulties among residents and prevalence of hearing loss in this population is underreported. This can result in an overestimation of levels of cognitive impairment. Untreated hearing loss is associated with social isolation, depression, disruptive behaviour and cognitive decline. This study aimed to explore the provision of hearing care (hearing assessment, rehabilitation and staff training) in Scottish care homes for older people.

Methods. A survey comprising 18 questions was distributed to the managers (or designated staff members) of 659 care homes across Scotland.

Results. Responses were obtained from 154 care homes. The results support existing evidence that hearing is not assessed in the majority of homes, resulting in under detection of hearing loss. Staff lack training in supporting residents' hearing needs. Access to hearing care in residential homes differs across health board areas.

Conclusions. There is an urgent requirement for hearing assessment of older adults on admission to residential care. Care providers require this information to construct effective care plans and mitigate the effects of hearing loss. Those responsible for providing hearing rehabilitation services require information about service users to address any unmet need. 
Introduction

The population of older people in care homes, many with on-going multimorbidities and increasingly complex needs, is projected to rise ${ }^{1,2}$. According to the NHS Scottish Care Home Census there are currently over 31,000 older people in Scottish care homes ${ }^{3}$. It is estimated that over $89 \%$ currently have some level of cognitive impairment, up to $75 \%$ are frail and may be immobile or require assistance with mobility and at least $40 \%$ experience depression $4,5,6$.

The presence of undiagnosed and untreated hearing loss can mean that caregivers mistake the symptoms of hearing loss for cognitive dysfunction ${ }^{7}$ and it is known that dementia is significantly over-estimated in adults with untreated hearing loss ${ }^{8}$. Recent research has also found that hearing loss is significantly associated with an increased risk of dementia ${ }^{9,10}$. Both hearing loss and lower cognitive status can also be associated with disruptive behaviour within residential homes ${ }^{11,12}$.

The majority of older adults who enter residential care will remain until death and the average length of stay is just over 2 years ${ }^{3}$. It is of vital importance to the resident, their families and friends that they are able to engage in meaningful conversations during this time. It is also essential that older adults are able to plan their care in advance as well as discuss end of life care requirements with their care givers ${ }^{16,17}$. The Scottish Government's See Hear strategy states that there should be seamless provision of assessment, care and support for adults with hearing impairment ${ }^{18}$.

The current NICE guideline for assessment and management of Hearing Loss in Adults identifies the need for research into hearing loss prevalence of people who under-present for hearing loss 19. The prevalence of hearing loss is known to increase with age and in adults over the age of 70 is estimated to be $76 \%{ }^{7}$. Indeed, the prevalence of hearing loss in adults living in care homes has been proposed to be in excess of $90 \%{ }^{20}$. It is suggested that older people with hearing loss are more likely to require residential care due to associated higher levels of cognitive impairment, including reduced processing speed, in addition to lower reserves available for listening effort. 
Older adults, whether or not they have a hearing loss, may have difficulties with the processing of auditory information, particularly in challenging listening environments ${ }^{13,14}$. In the care home environment, high levels of environmental noise impact negatively on residents' communication 15. According to the Scottish Care Home Census the average age of those living in Scottish care homes for older adults is 84 years and yet the same report finds that only $9 \%$ of residents are identified as having hearing impairments ${ }^{3}$. This apparent disparity in expected prevalence versus reported prevalence requires urgent investigation. The authors conducted an online survey of care home managers across Scotland to explore the current situation in relation to hearing assessment, aural rehabilitation and staff training.

Method

- Data collection

The survey was developed by a team of academics at Queen Margaret University with experience in audiology, hearing therapy, speech and language therapy, psychology, counselling and linguistics. Questions were selected on the basis of a literature review, discussions with residential care home managers and nursing staff, and our own experiences of providing hearing care for older people in residential care. A first draft of the survey was reviewed by care home staff and revisions were made, based on their feedback. These revisions were primarily aimed at simplifying questions to increase the likelihood of survey completion.

The survey comprised 18 questions, addressing the following: geographical location; numbers of residents; the provision of hearing assessments; hearing aid use; cerumen management; aids to communication; staff training. Questions used either multiple choice format or asked for short typed answers. A copy of the survey can be found in the appendix.

Of the care homes across Scotland, 931 were identified as providing residential care for older people. Contact email addresses were obtained for 659 (71\%) of these homes. All 659 homes were sent an email, for the attention of the care home manager or a designated member of staff, with a weblink to the electronic survey. Reminder emails were sent at 5 and 10 weeks after the initial invitation. 
- Data analysis

Responses were analysed using basic descriptive statistics and reported by themes.

Results

Responses were received from care homes in every Scottish health board area. A total of 154 survey responses were received. This represents $23 \%$ of the care homes contacted, although the response rate varied from $10 \%$ to $50 \%$ across different health boards. Respondents reported that they were caring for a total of 5351 older adults, of whom 3216 required nursing care and this equates to $17 \%$ of the total population of long stay residents in care homes for older people in Scotland. Figure 1 shows the distribution of care homes participating in the survey according to their health board area.

\section{INSERT FIGURE 1.}

Figure 2. represents the percentage of respondents from each health board area who reported routinely testing residents' hearing on admission to the care home. These results should be viewed in the context of the wide variability in response rates from different health board areas. For example, only one care home manager in the Western Isles (out of 7 who received the survey) responded and the $100 \%$ figure therefore represents only one care home routinely testing adults on admission. By contrast, 23 care home managers in Greater Glasgow and Clyde responded (out of 139 who received the survey) and only 3 (13\%) of those managers reported that residents had their hearing tested on admission.

\section{INSERT FIGURE 2.}


While $88 \%$ of respondents reported that their care home did not routinely assess residents' hearing on entry to the home, $100 \%$ of care homes stated that a section on communication needs was included in each resident's care plan. In those care homes where routine assessment did take place, $47 \%$ were carried out by NHS audiologists. The remainder were carried out either by care home staff, GP practice staff or private practitioners. No details were obtained about the nature of these assessments.

The majority (86\%) of care homes reported that, should an existing resident experience hearing difficulties, their GP would be contacted. In $9 \%$ of care homes, the resident would be referred directly to their local NHS Audiology service. In $75 \%$ of cases, a resident requiring a hearing assessment would have to travel to another location for assessment.

On average, respondents reported that $22 \%$ of their older residents wore hearing aids. This figure ranged from 5\%-30\% across different health board areas, with the exception of Orkney, where 10 out of 11 residents $(91 \%)$ wore hearing aids. Of the residents who used hearing aids, $79 \%$ were reported to be wearing their hearing aids every day. The majority of hearing aid wearers $(80 \%)$ needed assistance putting in their hearing aids, while $91 \%$ needed assistance with maintenance tasks, including changing batteries and cleaning. However, on average only $40 \%$ of care homes reported that staff had received any formal training in the care and maintenance of hearing aids. This figure ranged from 0\%-80\% across different health board areas. The variability across health board areas is illustrated in figure 3. It is noted that there is no obvious correspondence between those care homes offering routine hearing testing and those offering staff training on aspects of hearing care.

\section{INSERT FIGURE 3}

Respondents were asked about the availability and use of a range of assistive listening devices and other aids to support communication. These included: loop amplifier (telecoil) systems; soundfield systems with speakers; TV listening headsets; FM systems; flashing light alert systems for fire alarms; flashing light alert systems to alert residents to e.g. doorbells, telephones, vibrating alert system; picture-based communication systems; internet and email facilities; video 
telephones; visual message boards. Respondents were given the option to describe any other communication aids in use. In $47 \%$ of participating care homes, these devices were not provided within residents' own rooms. The most commonly provided devices within rooms were picturebased communication systems (24\% of homes) and TV listening headsets (19\% of homes). In $89 \%$ of care homes some form of assistive listening devices or other aids to support communication were provided within communal areas. The most commonly used communication aids were visual message boards (56\% of homes) and picture-based communication systems (45\% of homes). In $40 \%$ of participating homes, internet and email facilities were available in communal areas. The most commonly used assistive listening devices were loop amplifier (telecoil) systems, which were used in communal areas (29\% of homes). The use of other devices was very limited. Only $27 \%$ of respondents reported staff in their care home having received formal training in the use of communication aids. Around 50\% of respondents reported that staff had received formal training in strategies for communicating with adults who are hearing impaired. In around a third of cases, training in communication strategies and the use of communication aids was reported to have been provided by care home staff.

\section{Discussion}

Main finding of this study

Although there is a section on communication needs in each resident's care plan, the majority of care homes do not have knowledge of their residents' hearing thresholds. On average, managers reported that only $22 \%$ of their older residents had hearing aids. While this figure is higher than suggested by the Scottish Care Homes Census, there is extensive evidence that the prevalence of hearing impairment in older adults is far greater than these findings would indicate ${ }^{3,7}$. One of the more common assistive listening devices provided in the communal areas were loop amplifier (telecoil) systems, however these require the residents to be wearing hearing aids with an activated telecoil system. It can be inferred that older adults in residential care in Scotland are under-presenting for hearing loss and that there is inequality of provision of assessment, care and support for adults with hearing impairment.

What is already known on this topic 
There is a growing body of evidence that untreated hearing loss in adults is a risk factor for increased distress, depression, loneliness, paranoia, cognitive decline and dementia ${ }^{10,17}$. There is an urgent requirement for those who provide care for older adults, as well as those responsible for providing hearing rehabilitation services, to investigate ways of addressing the hearing care needs of this population ${ }^{21,22}$.

There appears to be a disconnect between communication needs, hearing care provision and staff training. On average, $40 \%$ of staff have received training regarding hearing aid care and maintenance and this is a consistent finding in the literature. In a previous study around half of care home staff were uncertain as to how to change a hearing aid battery and had not received training on hearing $\operatorname{loss}{ }^{15}$.

What this study adds

The high prevalence of hearing problems in this population would indicate a need for all older adults to have their hearing assessed when first entering a care home and at regular intervals thereafter. Routine hearing testing on entry to a care home was found to take place in fewer than $20 \%$ of the homes surveyed. Contributing factors may include difficulties accessing audiology services. NHS audiology services do not routinely provide hearing services for care home residents. In the majority of cases, it appears that referral pathways require residents to be referred to audiology through their own GP and then to travel to another location to have their hearing assessed. With $60 \%$ of residents requiring nursing care, this is likely to be a difficult and potentially distressing process for many. A standard adult hearing test uses Pure Tone Audiometry, which requires the patient to wear headphones and actively listen for, and respond to, tones presented at various frequencies and amplitudes. This task may well be less straightforward for older adults, particularly those with symptoms of dementia. More than one appointment and a customised test battery may be required for accurate results to be obtained.

Once a hearing loss is identified, treatment and rehabilitation is required. For most adults, treatment will begin with the fitting of a hearing aid. It is well established that as many as $24 \%$ of older adults fitted with hearing aids do not wear them and many cite difficulties in inserting and managing hearing aids as a key reason for non-use ${ }^{23,24}$. It is therefore a matter of some concern 
that, while care home managers report that $80 \%$ of residents fitted with hearing aids needed help inserting them and $91 \%$ needed help with care and maintenance, $60 \%$ of care homes report having had no formal staff training in this area.

Hearing aids are not the only rehabilitation option for adults with hearing impairment and it may be that the care home environment lends itself to the use of other approaches to aural rehabilitation ${ }^{24}$. Assistive listening devices and other aids to communication can be valuable for those who do not wear hearing aids, as well as for those who do. Only a minority of care homes were making use of many of these devices and again, there was a lack of training in their use.

\section{Limitations of this study}

The response rate across the different health board regions varied from 10 to $50 \%$. The survey relied on contact data regarding care home managers to be available and current. There is a high staff turnover in the care home environment with $27 \%$ of new employees leaving post within two years. There are also high vacancy rates $(9 \%)$ and high sickness absence rates $(6 \%)$ within the sector. It is acknowledged that the care home sector is under pressure and that response rates to surveys are falling ${ }^{25}$.

It is also acknowledged that the results reported here are based solely on the views of care home managers and do not capture the experiences of care home residents themselves. This is an area for future research.

\section{Conclusion}

Both within and between Scottish health board areas, a substantial inequality of access to hearing assessment and hearing care is evident from the findings of this survey. Hearing status remains unknown and hearing loss untreated in large numbers of older residents. The potential consequences of this, in relation to quality of life and care planning are severe. A more cohesive approach to hearing care is required for older adults in residential homes. Increased collaboration between audiology services and residential care providers is needed to address the identification and treatment of hearing loss, and the training of care home staff. 
Acknowledgements

We thank all participants for supporting this study.

Conflicts of interest

None declared.

References

1. National Records of Scotland. Projected Population of Scotland (2016-based). Edinburgh:

National Statistics, 2017. https://www.nrscotland.gov.uk/statistics-and-data/statistics/statistics-

by-theme/population/population-projections/population-projections-scotland/2016-based

(25 October 2018, date last accessed).

2. Green I, Stow D, Matthews FE et al. . Changes over time in the health and functioning of older people moving into care homes: analysis of data from the English Longitudinal Study of Ageing. Age Ageing 2017;46(4):693-6.

3. ISD Scotland. Care Home Census for Adults in Scotland. Scotland: Information Services Division (ISD), 2018. https://www.isdscotland.org/Health-Topics/Health-and-SocialCommunity-Care/Publications/2018-09-11/2018-09-11-CHCensus-Report.pdf?2721804381 (25 October 2018, date last accessed).

4. Scottish Government. Annual Report of the Chief Medical Officer. Edinburgh: Scottish Government, 2009.

5. Kojima G. Prevalence of Frailty in Nursing Homes: A Systematic Review and Meta-Analysis. J Am Med Dir Assoc 2015;16(11):940-5.

6. Morrell CJ, Curran S, Topping A et al. . Identification of depressive disorder among older people in care homes - a feasibility study. Prim Health Care Res Dev 2011;12(3):255-265. 
7. Yamasoba T, Lin FR, Someya $S$ et al. .Current concepts in age-related hearing loss: epidemiology and mechanistic pathways. Hear Res 2013;303:30-8.

8. Jorgensen LE, Palmer CV, Pratt S et al. .The Effect of Decreased Audibility on MMSE Performance: A Measure Commonly Used for Diagnosing Dementia. J Am Acad Audiol 2016; 27(4):311-23.

9. Lin FR, Metter E J, O'Brien R J, et al. .Hearing Loss and Incident Dementia. Arch Neurol 2011;6(2):214-220.

10. Lin FR, Yaffe K, Xia J. et al. .Hearing loss and cognitive decline in older adults. JAMA Intern Med 2013;173(4):293-299.

11. Hall KA, O'Connor DW.. Correlates of Aggressive Behavior in Dementia. Int Psychogeriatr 2004;16(2):141-58.

12. Robitaille A, Garcia L, McIntosh C. Joint trajectories of cognitive functioning and challenging behavior for persons living with dementia in long-term care. Psychol Aging. 2015;30(3):712-26.

13. Kortlang S, Mauermann M, Ewert SD. Suprathreshold auditory processing deficits in noise: Effects of hearing loss and age. Hear Res 2016;331:27-40.

14. Li-Korotky HS. Age-related hearing loss: quality of care for quality of life. Gerontologist 2012;52(2):265-271.

15. Pryce H, Gooberman-Hill R. 'There's a hell of a noise': living with a hearing loss in residential care. Age Ageing, 2012;41(1):40-46.

16. Bailoor K, Kamil LH, Goldman E et al. The Voice Is As Mighty As the Pen: Integrating Conversations into Advance Care Planning. J Bioeth Inq 2018;15(2):185-191. 
17. Scottish Government. Reshaping Care for Older People - 'Getting on'. Edinburgh: Scottish Government, 2013.

18. Scottish Government. See Hear. A strategic framework for meeting the needs of people with a sensory impairment in Scotland. Edinburgh: Scottish Government, 2014.

19. NICE. Hearing loss in adults: assessment and management. London: NICE, 2018

20. Allen NH, Burns A, Newton V et al. The effects of improving hearing in dementia. Age Ageing 2003:32(2):189-193.

21. Hartley D, Rochtchina E, Newall P, et al. . Use of hearing aids and assistive listening devices in an older Australian population. J Am Acad Audiol 2010;21:642 - 653.

22. Punch R, Horstmanshof L. Hearing loss and its impact on residents in long term care facilities: A systematic review of literature. Geriatr Nurs 2019;40(2):138-147.

23. McCormack A, Fortnum H. Why do people fitted with hearing aids not wear them? Int $J$ Audiol 2013;52(5):360-8.

24. Hickson L. The Challenge of Older People Living in Aged Care Environments. Chapter 11 in Proceedings of the Second International Adult Conference: The Challenge of Ageing, Chicago. Phonak: Switzerland, 2009.

25. National Care Forum. 2017 Personnel Statistics Benchmarking Survey Report. Coventry: NCF, 2017. http://www.nationalcareforum.org.uk/personnel-statistics-survey.asp?menu_ID=53 (25 October 2018, date last accessed). 